\title{
Integrable Discretization of the Coupled Nonlinear Schrödinger Equations
}

\author{
Takayuki Tsuchida* \\ Department of Physics, Graduate School of Science, \\ University of Tokyo, Hongo 7-3-1, Bunkyo-ku, \\ Tokyo 113-0033, Japan \\ tsuchida@monet.phys.s.u-tokyo.ac.jp
}

October 29, 2018

\begin{abstract}
A discrete version of the inverse scattering method proposed by Ablowitz and Ladik is generalized to study an integrable full-discretization (discrete time and discrete space) of the coupled nonlinear Schrödinger equations. The generalization enables one to solve the initial-value problem. Soliton solutions and conserved quantities of the full-discrete system are constructed.
\end{abstract}

\section{Introduction}

Among various soliton equations, a system of coupled nonlinear Schrödinger (CNLS) equations,

$$
\begin{aligned}
& \mathrm{i} \frac{\partial q_{j}}{\partial t}+\frac{\partial^{2} q_{j}}{\partial x^{2}}-2 \sum_{k=1}^{m} q_{k} r_{k} \cdot q_{j}=0 \\
& \mathrm{i} \frac{\partial r_{j}}{\partial t}-\frac{\partial^{2} r_{j}}{\partial x^{2}}+2 \sum_{k=1}^{m} r_{k} q_{k} \cdot r_{j}=0
\end{aligned}
$$

is physically significant since it describes multi-mode wave propagation in nonlinear optics. The CNLS equations can be solved via the inverse scattering method (ISM) under the reduction $r_{j}=$ $-q_{j}^{*}$ and the decaying boundary conditions $q_{j} \rightarrow 0$ as $x \rightarrow \pm \infty$. It is, however, not always easy to trace time evolution of initial values for soliton equations solvable via the ISM. Thus, it is an important problem to find good schemes for numerical computation of soliton equations. One possible approach is to find discretization of soliton equations with preserving the complete integrability (integrable discretization for short). The discretized soliton equations are not only useful for numerical computation but also interesting as models of nonlinear population dynamics, nonlinear electric circuits and nonlinear lattice vibrations.

An integrable discretization of the nonlinear Schrödinger (NLS) equation ((11) with $m=1$ ) was proposed by Ablowitz and Ladik [2, 3]. The author, Ujino and Wadati generalized the formulation of Ablowitz and Ladik and found an integrable semi-discretization (space discretization) of the

\footnotetext{
* Supported by JSPS Research Fellowships for Young Scientists.
} 
coupled modified KdV equations [4] and the CNLS equations [5]. In this article, as a continuation of [5], we consider an integrable discretization of both space and time (full-discretization for short) for the CNLS equations (1). In accordance with the page limit, we concisely explain an outline of the ISM for the model. The reader can refer to [4, 5 for more details because we can perform the ISM in the same manner as in these papers, apart from the difference in time dependences of scattering data.

\section{Lax pair}

We introduce a set of auxiliary linear equations,

$$
\Psi_{n+1}=L_{n} \Psi_{n}, \quad \tilde{\Psi}_{n}=V_{n} \Psi_{n},
$$

where the tilde $(\sim)$ denotes the time shift in discrete time $l \in \mathbb{Z}(l \rightarrow l+1)$. The compatibility condition of (2) is given by

$$
\tilde{L}_{n} V_{n}=V_{n+1} L_{n} .
$$

$L_{n}$ and $V_{n}$, and (3) are respectively the Lax pair and the zero-curvature condition (or Lax equation) in the full-discrete case. To obtain a full-discrete version of the CNLS equations, we choose the form of the Lax pair as

$$
\begin{aligned}
& L_{n}=z\left[\begin{array}{cc}
\mathrm{e}^{\mathrm{i} \alpha H_{1}} & \\
& O
\end{array}\right]+\left[\begin{array}{cc}
O & \mathrm{e}^{\mathrm{i} \alpha H_{1}} \mathcal{Q}_{n} \\
\mathrm{e}^{\mathrm{i} \alpha H_{2}} \mathcal{R}_{n} & O
\end{array}\right]+\frac{1}{z}\left[\begin{array}{cc}
O & \\
& \mathrm{e}^{\mathrm{i} \alpha H_{2}}
\end{array}\right], \\
& V_{n}=\left[\begin{array}{ll}
\mathrm{e}^{\mathrm{i} \beta H_{1}} & \\
& \mathrm{e}^{\mathrm{i} \beta H_{2}}
\end{array}\right]+\frac{\sin \beta}{\sin (2 \alpha+\beta)} j_{n}\left\{z^{2}\left[\begin{array}{ll}
O & \\
& -\mathrm{e}^{\mathrm{i} \beta H_{2}}
\end{array}\right]\right. \\
& +z\left[\begin{array}{cc}
O & \tilde{\mathcal{Q}}_{n} \mathrm{e}^{\mathrm{i} \beta H_{2}} \\
\mathrm{e}^{\mathrm{i}(\alpha+\beta) H_{2}} \mathcal{R}_{n-1} \mathrm{e}^{-\mathrm{i} \alpha H_{1}} & O
\end{array}\right] \\
& +\left[\begin{array}{ll}
-\tilde{\mathcal{Q}}_{n} \mathrm{e}^{\mathrm{i}(\alpha+\beta) H_{2}} \mathcal{R}_{n-1} \mathrm{e}^{-\mathrm{i} \alpha H_{1}} & \\
& -\tilde{\mathcal{R}}_{n} \mathrm{e}^{\mathrm{i}(\alpha+\beta) H_{1}} \mathcal{Q}_{n-1} \mathrm{e}^{-\mathrm{i} \alpha H_{2}}
\end{array}\right] \\
& \left.+\frac{1}{z}\left[\begin{array}{cc}
O & \mathrm{e}^{\mathrm{i}(\alpha+\beta) H_{1}} \mathcal{Q}_{n-1} \mathrm{e}^{-\mathrm{i} \alpha H_{2}} \\
\tilde{\mathcal{R}}_{n} \mathrm{e}^{\mathrm{i} \beta H_{1}} & O
\end{array}\right]+\frac{1}{z^{2}}\left[\begin{array}{ll}
-\mathrm{e}^{\mathrm{i} \beta H_{1}} & \\
& O
\end{array}\right]\right\} .
\end{aligned}
$$

Here $z$ is the spectral parameter which is time-independent. $\mathcal{Q}_{n}$ and $\mathcal{R}_{n}$ are $p \times p$ matrices. $H_{1}$ and $H_{2}$ are $p \times p$ constant matrices. $\alpha$ and $\beta$ are constants. $j_{n}$ is a scalar variable. We assume the following relations,

$$
\begin{gathered}
{\left[H_{1}, \mathcal{Q}_{n} \mathcal{R}_{n}\right]=\left[H_{2}, \mathcal{R}_{n} \mathcal{Q}_{n}\right]=O,} \\
H_{1} \mathcal{Q}_{n}-\mathcal{Q}_{n} H_{2}=-2 F_{1} \mathcal{Q}_{n} F_{2}, \quad H_{2} \mathcal{R}_{n}-\mathcal{R}_{n} H_{1}=2 F_{2} \mathcal{R}_{n} F_{1},
\end{gathered}
$$

where $[\cdot, \cdot]$ denotes the commutator and $F_{1}, F_{2}$ are $p \times p$ constant matrices which satisfy

$$
\left(F_{1}\right)^{2}=\left(F_{2}\right)^{2}=I, \quad\left[F_{1}, H_{1}\right]=\left[F_{2}, H_{2}\right]=O .
$$

Here $I$ is the identity matrix. We notice that the following relations hold:

$$
\begin{aligned}
\mathrm{e}^{-\mathrm{i} y H_{1}} \mathcal{Q}_{n} \mathrm{e}^{\mathrm{i} y H_{2}} & =\cos (2 y) \mathcal{Q}_{n}+\mathrm{i} \sin (2 y) F_{1} \mathcal{Q}_{n} F_{2}, \\
\mathrm{e}^{\mathrm{i} y H_{2}} \mathcal{R}_{n} \mathrm{e}^{-\mathrm{i} y H_{1}} & =\cos (2 y) \mathcal{R}_{n}+\mathrm{i} \sin (2 y) F_{2} \mathcal{R}_{n} F_{1} .
\end{aligned}
$$


We write the difference interval of time by $\delta t$. Putting (4) and (5) into (3), we obtain

$$
\begin{aligned}
& \mathrm{i} \frac{1}{\delta t}\left(\tilde{\mathcal{Q}}_{n}-\mathcal{Q}_{n}\right)-\mathrm{i} \frac{\tan \beta \cot (2 \alpha+\beta)}{\delta t}\left\{j_{n+1} \tilde{\mathcal{Q}}_{n+1}\left(I-\mathcal{R}_{n} \mathcal{Q}_{n}\right)-j_{n+1}\left(I-\mathcal{Q}_{n} \mathcal{R}_{n}\right) \mathcal{Q}_{n-1}\right\} \\
& +\frac{\tan \beta}{\delta t}\left\{j_{n+1} F_{1} \tilde{\mathcal{Q}}_{n+1} F_{2}\left(I-\mathcal{R}_{n} \mathcal{Q}_{n}\right)+j_{n+1}\left(I-\mathcal{Q}_{n} \mathcal{R}_{n}\right) F_{1} \mathcal{Q}_{n-1} F_{2}\right. \\
& \left.-F_{1}\left(\tilde{\mathcal{Q}}_{n}+\mathcal{Q}_{n}\right) F_{2}\right\}=O, \\
& \mathrm{i} \frac{1}{\delta t}\left(\tilde{\mathcal{R}}_{n}-\mathcal{R}_{n}\right)-\mathrm{i} \frac{\tan \beta \cot (2 \alpha+\beta)}{\delta t}\left\{j_{n+1} \tilde{\mathcal{R}}_{n+1}\left(I-\mathcal{Q}_{n} \mathcal{R}_{n}\right)-j_{n+1}\left(I-\mathcal{R}_{n} \mathcal{Q}_{n}\right) \mathcal{R}_{n-1}\right\} \\
& -\frac{\tan \beta}{\delta t}\left\{j_{n+1} F_{2} \tilde{\mathcal{R}}_{n+1} F_{1}\left(I-\mathcal{Q}_{n} \mathcal{R}_{n}\right)+j_{n+1}\left(I-\mathcal{R}_{n} \mathcal{Q}_{n}\right) F_{2} \mathcal{R}_{n-1} F_{1}\right. \\
& \left.-F_{2}\left(\tilde{\mathcal{R}}_{n}+\mathcal{R}_{n}\right) F_{1}\right\}=O, \\
& j_{n+1}\left(I-\mathcal{Q}_{n} \mathcal{R}_{n}\right)=j_{n}\left(I-\tilde{\mathcal{Q}}_{n} \tilde{\mathcal{R}}_{n}\right), \\
& j_{n+1}\left(I-\mathcal{R}_{n} \mathcal{Q}_{n}\right)=j_{n}\left(I-\tilde{\mathcal{R}}_{n} \tilde{\mathcal{Q}}_{n}\right),
\end{aligned}
$$

with the help of (9). If we set

$$
2 \alpha+\beta=\frac{\pi}{2}, \quad \frac{\tan \beta}{\delta t}=1,
$$

or equivalently,

$$
\alpha=\frac{\pi}{4}-\frac{1}{2} \tan ^{-1} \delta t, \quad \beta=\tan ^{-1} \delta t,
$$

(10a) and (10b) are simplified as

$$
\begin{aligned}
& \mathrm{i} \frac{1}{\delta t}\left(\tilde{\mathcal{Q}}_{n}-\mathcal{Q}_{n}\right)+j_{n+1} F_{1} \tilde{\mathcal{Q}}_{n+1} F_{2}\left(I-\mathcal{R}_{n} \mathcal{Q}_{n}\right)+j_{n+1}\left(I-\mathcal{Q}_{n} \mathcal{R}_{n}\right) F_{1} \mathcal{Q}_{n-1} F_{2} \\
& \quad-F_{1}\left(\tilde{\mathcal{Q}}_{n}+\mathcal{Q}_{n}\right) F_{2}=O \\
& \mathrm{i} \frac{1}{\delta t}\left(\tilde{\mathcal{R}}_{n}-\mathcal{R}_{n}\right)-j_{n+1} F_{2} \tilde{\mathcal{R}}_{n+1} F_{1}\left(I-\mathcal{Q}_{n} \mathcal{R}_{n}\right)-j_{n+1}\left(I-\mathcal{R}_{n} \mathcal{Q}_{n}\right) F_{2} \mathcal{R}_{n-1} F_{1} \\
& \quad+F_{2}\left(\tilde{\mathcal{R}}_{n}+\mathcal{R}_{n}\right) F_{1}=O .
\end{aligned}
$$

To consider a reduction of these matrix equations to full-discrete CNLS equations, we define $F_{1}$, $F_{2}, H_{1}, H_{2}, \mathcal{Q}_{n}$ and $\mathcal{R}_{n}$ recursively as follows:

$$
\begin{gathered}
F_{1}^{(1)}=1, \quad F_{2}^{(1)}=1, \quad H_{1}^{(1)}=-1, \quad H_{2}^{(1)}=1, \quad \mathcal{Q}_{n}^{(1)}=q_{n}^{(1)}, \quad \mathcal{R}_{n}^{(1)}=r_{n}^{(1)}, \\
F_{1}^{(m+1)}=\left[\begin{array}{rr}
F_{1}^{(m)} & -F_{2}^{(m)}
\end{array}\right], \quad F_{2}^{(m+1)}=\left[\begin{array}{rr}
F_{2}^{(m)} & F_{1}^{(m)}
\end{array}\right], \\
H_{1}^{(m+1)}=\left[\begin{array}{rr}
H_{1}^{(m)}-I_{2^{m-1}} & H_{2}^{(m)}+I_{2^{m-1}}
\end{array}\right], \\
H_{2}^{(m+1)}=\left[\begin{array}{rr}
H_{2}^{(m)}-I_{2^{m-1}} & H_{1}^{(m)}+I_{2^{m-1}}
\end{array}\right], \\
\mathcal{Q}_{n}^{(m+1)}=\left[\begin{array}{cc}
\mathcal{Q}_{n}^{(m)} & q_{n}^{(m+1)} I_{2^{m-1}}^{(m)} \\
r_{n}^{(m+1)} I_{2^{m-1}} & -\mathcal{R}_{n}^{(m)}
\end{array}\right], \quad \mathcal{R}_{n}^{(m+1)}=\left[\begin{array}{cc}
\mathcal{R}_{n}^{(m)} & q_{n}^{(m+1)} I_{2^{m-1}} \\
r_{n}^{(m+1)} I_{2^{m-1}} & -\mathcal{Q}_{n}^{(m)}
\end{array}\right] .
\end{gathered}
$$


Here $I_{2^{m-1}}$ is the $2^{m-1} \times 2^{m-1}$ identity matrix. We can easily show that (6)-(8) and the relation,

$$
Q_{n}^{(m)} R_{n}^{(m)}=R_{n}^{(m)} Q_{n}^{(m)}=\sum_{k=1}^{m} q_{n}^{(k)} r_{n}^{(k)} \cdot I_{2^{m-1}},
$$

are satisfied. Then it is straightforward to prove that substitution of $F_{1}^{(m)}, F_{2}^{(m)}, \mathcal{Q}_{n}^{(m)}$ and $\mathcal{R}_{n}^{(m)}$ into (10d), (10d), (11a) and (11b) yields the following full-discrete CNLS equations,

$$
\begin{gathered}
\mathrm{i} \frac{1}{\delta t}\left(\tilde{q}_{n}^{(j)}-q_{n}^{(j)}\right)+\left(1-\sum_{k=1}^{m} q_{n}^{(k)} r_{n}^{(k)}\right) j_{n+1}\left(\tilde{q}_{n+1}^{(j)}+q_{n-1}^{(j)}\right)-\left(\tilde{q}_{n}^{(j)}+q_{n}^{(j)}\right)=0, \\
\mathrm{i} \frac{1}{\delta t}\left(\tilde{r}_{n}^{(j)}-r_{n}^{(j)}\right)-\left(1-\sum_{k=1}^{m} r_{n}^{(k)} q_{n}^{(k)}\right) j_{n+1}\left(\tilde{r}_{n+1}^{(j)}+r_{n-1}^{(j)}\right)+\left(\tilde{r}_{n}^{(j)}+r_{n}^{(j)}\right)=0, \\
j_{n+1}\left(1-\sum_{k=1}^{m} q_{n}^{(k)} r_{n}^{(k)}\right)=j_{n}\left(1-\sum_{k=1}^{m} \tilde{q}_{n}^{(k)} \tilde{r}_{n}^{(k)}\right), \quad j=1,2, \ldots, m .
\end{gathered}
$$

Assuming the reduction $r_{n}^{(j)}=-q_{n}^{(j) *}$, we obtain the self-focusing case of the full-discrete CNLS equations:

$$
\begin{gathered}
\mathrm{i} \frac{1}{\delta t}\left(\tilde{q}_{n}^{(j)}-q_{n}^{(j)}\right)+\left(1+\sum_{k=1}^{m}\left|q_{n}^{(k)}\right|^{2}\right) j_{n+1}\left(\tilde{q}_{n+1}^{(j)}+q_{n-1}^{(j)}\right)-\left(\tilde{q}_{n}^{(j)}+q_{n}^{(j)}\right)=0 \\
j_{n+1}\left(1+\sum_{k=1}^{m}\left|q_{n}^{(k)}\right|^{2}\right)=j_{n}\left(1+\sum_{k=1}^{m}\left|\tilde{q}_{n}^{(k)}\right|^{2}\right), \quad j=1,2, \ldots, m .
\end{gathered}
$$

In the one-component case $(m=1)$, this system coincides with the full-discrete NLS equation obtained by Ablowitz and Ladik [3]. If we take the continuum limit of time $(\delta t \rightarrow 0)$, we can equate $j_{n}$ to 1 . Thus, the system (12) reduces to the semi-discrete CNLS equations [5],

$$
\begin{aligned}
& \mathrm{i} \frac{\partial q_{n}^{(j)}}{\partial t}+\left(q_{n+1}^{(j)}+q_{n-1}^{(j)}-2 q_{n}^{(j)}\right)-\sum_{k=1}^{m} q_{n}^{(k)} r_{n}^{(k)} \cdot\left(q_{n+1}^{(j)}+q_{n-1}^{(j)}\right)=0, \\
& \mathrm{i} \frac{\partial r_{n}^{(j)}}{\partial t}-\left(r_{n+1}^{(j)}+r_{n-1}^{(j)}-2 r_{n}^{(j)}\right)+\sum_{k=1}^{m} r_{n}^{(k)} q_{n}^{(k)} \cdot\left(r_{n+1}^{(j)}+r_{n-1}^{(j)}\right)=0,
\end{aligned}
$$

The corresponding linear problem is given by taking the continuum limit:

$$
\Psi_{n+1}=L_{n} \Psi_{n}, \quad \frac{\partial \Psi_{n}}{\partial t}=M_{n} \Psi_{n},
$$

with

$$
\begin{aligned}
L_{n}=z & {\left[\begin{array}{ll}
\mathrm{e}^{\mathrm{i} \frac{\pi}{4} H_{1}} & \\
& O
\end{array}\right]+\left[\begin{array}{cc}
O & \mathrm{e}^{\mathrm{i} \frac{\pi}{4} H_{1}} \mathcal{Q}_{n} \\
\mathrm{e}^{\mathrm{i} \frac{\pi}{4} H_{2}} \mathcal{R}_{n} & O
\end{array}\right]+\frac{1}{z}\left[\begin{array}{cc}
O & \\
& \mathrm{e}^{\mathrm{i} \frac{\pi}{4} H_{2}}
\end{array}\right], } \\
M_{n}= & z^{2}\left[\begin{array}{cc}
O & \\
& -I
\end{array}\right]+z\left[\begin{array}{cc}
\mathrm{e}^{\mathrm{i} \frac{\pi}{4} H_{2}} \mathcal{R}_{n-1} \mathrm{e}^{-\mathrm{i} \frac{\pi}{4} H_{1}} & O
\end{array}\right] \\
& +\left[\begin{array}{cc}
-\mathcal{Q}_{n} \mathrm{e}^{\mathrm{i} \frac{\pi}{4} H_{2}} \mathcal{R}_{n-1} \mathrm{e}^{-\mathrm{i} \frac{\pi}{4} H_{1}}+\mathrm{i} H_{1} & -\mathcal{R}_{n} \mathrm{e}^{\mathrm{i} \frac{\pi}{4} H_{1}} \mathcal{Q}_{n-1} \mathrm{e}^{-\mathrm{i} \frac{\pi}{4} H_{2}}+\mathrm{i} H_{2}
\end{array}\right] \\
& +\frac{1}{z}\left[\begin{array}{cc}
O & \mathrm{e}^{\mathrm{i} \frac{\pi}{4} H_{1}} \mathcal{Q}_{n-1} \mathrm{e}^{-\mathrm{i} \frac{\pi}{4} H_{2}} \\
\mathcal{R}_{n} & O
\end{array}\right]+\frac{1}{z^{2}}\left[\begin{array}{cc}
-I & \\
& O
\end{array}\right] .
\end{aligned}
$$


It should be noted that the above Lax pair for the semi-discrete CNLS equations is equivalent to the one given in [5] via a gauge transformation.

\section{Inverse scattering method}

As was shown in the previous section, the linear problem for the full-discrete CNLS equations (12) is expressed in terms of a $2^{m} \times 2^{m}$ matrix as

$$
\left[\begin{array}{l}
\Psi_{1 n+1} \\
\Psi_{2 n+1}
\end{array}\right]=\left[\begin{array}{cc}
z \mathrm{e}^{\mathrm{i} \alpha H_{1}^{(m)}} & \mathrm{e}^{\mathrm{i} \alpha H_{1}^{(m)}} \mathcal{Q}_{n}^{(m)} \\
\mathrm{e}^{\mathrm{i} \alpha H_{2}^{(m)}} \mathcal{R}_{n}^{(m)} & \frac{1}{z} \mathrm{e}^{\mathrm{i} \alpha H_{2}^{(m)}}
\end{array}\right]\left[\begin{array}{c}
\Psi_{1 n} \\
\Psi_{2 n}
\end{array}\right]
$$

where $\alpha=\frac{\pi}{4}-\frac{1}{2} \tan ^{-1} \delta t$. To simplify the analysis, we introduce a gauge transformation ( $l$ : discrete time),

$$
\left[\begin{array}{l}
\Phi_{1 n} \\
\Phi_{2 n}
\end{array}\right]=\left[\begin{array}{ll}
\mathrm{e}^{-\mathrm{i}(n \alpha+l \beta) H_{1}^{(m)}} & \\
& \mathrm{e}^{-\mathrm{i}(n \alpha+l \beta) H_{2}^{(m)}}
\end{array}\right]\left[\begin{array}{l}
\Psi_{1 n} \\
\Psi_{2 n}
\end{array}\right], \quad \beta=\tan ^{-1} \delta t,
$$

by which the linear problem (14) is changed into the standard form,

$$
\left[\begin{array}{l}
\Phi_{1 n+1} \\
\Phi_{2 n+1}
\end{array}\right]=\left[\begin{array}{cc}
z I & Q_{n}^{(m)} \\
R_{n}^{(m)} & \frac{1}{z} I
\end{array}\right]\left[\begin{array}{l}
\Phi_{1 n} \\
\Phi_{2 n}
\end{array}\right] .
$$

Here the transformed potentials are given by

$$
\begin{aligned}
Q_{n}^{(m)} & =\mathrm{e}^{-\mathrm{i}(n \alpha+l \beta) H_{1}^{(m)}} \mathcal{Q}_{n}^{(m)} \mathrm{e}^{\mathrm{i}(n \alpha+l \beta) H_{2}^{(m)},}, \\
R_{n}^{(m)} & =\mathrm{e}^{-\mathrm{i}(n \alpha+l \beta) H_{2}^{(m)}} \mathcal{R}_{n}^{(m)} \mathrm{e}^{\mathrm{i}(n \alpha+l \beta) H_{1}^{(m)}} .
\end{aligned}
$$

If we set

$$
\begin{aligned}
& \mathrm{e}^{2 \mathrm{i}(n \alpha+l \beta)} q_{n}^{(j)}=v_{n}^{(2 j-2)}+\mathrm{i} v_{n}^{(2 j-1)}, \\
& \mathrm{e}^{-2 \mathrm{i}(n \alpha+l \beta)} r_{n}^{(j)}=-v_{n}^{(2 j-2)}+\mathrm{i} v_{n}^{(2 j-1)}, \quad j=1,2, \ldots, m,
\end{aligned}
$$

$Q_{n}^{(m)}$ and $R_{n}^{(m)}$ for $m \geq 2$ are expressed as

$$
Q_{n}^{(m)}=v_{n}^{(0)} \mathbb{I}+\sum_{k=1}^{2 m-1} v_{n}^{(k)} e_{k}, \quad R_{n}^{(m)}=-v_{n}^{(0)} \mathbb{I}+\sum_{k=1}^{2 m-1} v_{n}^{(k)} e_{k} .
$$

Here $2^{m-1} \times 2^{m-1}$ matrices $\left\{e_{1}, \cdots, e_{2 m-1}\right\}$ satisfy the following important relations:

$$
\begin{aligned}
\left\{e_{i}, e_{j}\right\}_{+} & =-2 \delta_{i j} \mathbb{I}, \\
e_{k}^{\dagger} & =-e_{k},
\end{aligned}
$$

where $\{\cdot, \cdot\}_{+}$denotes the anticommutator and $\mathbb{I}$ is the $2^{m-1} \times 2^{m-1}$ unit matrix.

It should be remarked that the transformation (16) connects the full-discrete CNLS equations (12) with full-discrete coupled modified KdV equations [6, 8],

$$
\begin{gathered}
\frac{1}{\delta t}\left(\tilde{v}_{n}^{(j)}-v_{n}^{(j)}\right)=\left(1+\sum_{k=0}^{2 m-1} v_{n}^{(k) 2}\right) \Gamma_{n+1}\left(\tilde{v}_{n+1}^{(j)}-v_{n-1}^{(j)}\right), \quad j=0,1, \ldots, 2 m-1 \\
\Gamma_{n+1}\left(1+\sum_{k=0}^{2 m-1} v_{n}^{(k) 2}\right)=\Gamma_{n}\left(1+\sum_{k=0}^{2 m-1} \tilde{v}_{n}^{(k) 2}\right)
\end{gathered}
$$


where

$$
\Gamma_{n}=\frac{\sin \beta}{\delta t} j_{n} .
$$

We can investigate the direct and the inverse scattering problems associated with $(15)$ in the same way as in the semi-discrete case [4 5 by assuming $m \geq 2$, the reduction $r_{n}^{(j)}=-q_{n}^{(j) *}$ and the boundary conditions,

$$
q_{n}^{(j)}, r_{n}^{(j)} \rightarrow 0, \quad j_{n} \rightarrow \frac{\delta t}{\sin \beta} \quad \text { as } n \rightarrow \pm \infty
$$

In fact, $j_{n} \rightarrow$ const. $(n \rightarrow \pm \infty)$ is sufficient to perform the ISM. We have chosen the constant as (19) just for convenience.

We introduce Jost functions $\phi_{n}$ and $\bar{\phi}_{n}$ as solutions of (15) which satisfy the boundary conditions:

$$
\begin{aligned}
& \phi_{n} \sim\left[\begin{array}{c}
I \\
O
\end{array}\right] z^{n}, \quad \bar{\phi}_{n} \sim\left[\begin{array}{c}
O \\
-I
\end{array}\right] z^{-n} \quad \text { as } n \rightarrow-\infty, \\
& \phi_{n} \sim\left[\begin{array}{c}
A(z) z^{n} \\
B(z) z^{-n}
\end{array}\right], \quad \bar{\phi}_{n} \sim\left[\begin{array}{c}
\bar{B}(z) z^{n} \\
-\bar{A}(z) z^{-n}
\end{array}\right] \quad \text { as } \quad n \rightarrow+\infty .
\end{aligned}
$$

Here $\{A(z), \bar{A}(z), B(z), \bar{B}(z)\}$ are $n$-independent $2^{m-1} \times 2^{m-1}$ matrices which are called scattering data. Suppose that $1 / \operatorname{det} A(z)$ has $2 N$ isolated simple poles $\left\{z_{1}, z_{2}, \cdots, z_{2 N}\right\}$ in $|z|>1$ and is regular on the unit circle $C$. It will be explained why we choose the number of poles to be even. Let us define $F(n)$ in terms of the scattering data by

$$
F(n)=\frac{1}{2 \pi \mathrm{i}} \oint_{C} B(z) A(z)^{-1} z^{-n-1} \mathrm{~d} z+\sum_{j=1}^{2 N} C_{j} z_{j}^{-n-1},
$$

where $C_{j}$ is the residue matrix of $B(z) A(z)^{-1}$ at $z=z_{j}$. Similarly, we suppose that $1 / \operatorname{det} \bar{A}(z)$ has $2 \bar{N}$ isolated simple poles $\left\{\bar{z}_{1}, \bar{z}_{2}, \cdots, \bar{z}_{2 \bar{N}}\right\}$ in $|z|<1$ and is regular on the unit circle $C$. We define $\bar{F}(n)$ by

$$
\bar{F}(n)=\frac{1}{2 \pi \mathrm{i}} \oint_{C} \bar{B}(z) \bar{A}(z)^{-1} z^{n-1} \mathrm{~d} z-\sum_{k=1}^{2 \bar{N}} \bar{C}_{k} \bar{z}_{k}^{n-1},
$$

where $\bar{C}_{k}$ is the residue matrix of $\bar{B}(z) \bar{A}(z)^{-1}$ at $z=\bar{z}_{k}$. It can be shown after some computation that $F(n)$ and $\bar{F}(n)$ are connected with the physical variables $Q_{n}^{(m)}$ and $R_{n}^{(m)}$ through the following Gel'fand-Levitan-Marchenko equations:

$$
\begin{aligned}
& \kappa_{1}(n, q)=\bar{F}(n+q)-\sum_{n^{\prime}=n+1}^{\infty} \sum_{n^{\prime \prime}=n+1}^{\infty} \kappa_{1}\left(n, n^{\prime \prime}\right) F\left(n^{\prime \prime}+n^{\prime}\right) \bar{F}\left(n^{\prime}+q\right), \\
& \bar{\kappa}_{2}(n, q)=-F(n+q)-\sum_{n^{\prime}=n+1}^{\infty} \sum_{n^{\prime \prime}=n+1}^{\infty} \bar{\kappa}_{2}\left(n, n^{\prime \prime}\right) \bar{F}\left(n^{\prime \prime}+n^{\prime}\right) F\left(n^{\prime}+q\right),
\end{aligned}
$$

for $q>n$. Here

$$
-\kappa_{1}(n, n+1)=Q_{n}^{(m)}, \quad-\bar{\kappa}_{2}(n, n+1)=R_{n}^{(m)} .
$$

It should be noted that the scattering problem (15) gives the symmetry properties of the scattering data. Iterating (15), we can prove that $A(z), \bar{A}(z)$ are polynomials in $z$ of even degree 
and $B(z), \bar{B}(z)$ are polynomials in $z$ of odd degree. This fact gives the symmetry properties of $F$ and $\bar{F}$ :

$$
\begin{gathered}
F(n)=\left\{\begin{array}{cc}
2 F_{R}(n), & n: \text { odd }, \\
O, & n: \text { even, }
\end{array}\right. \\
\bar{F}(n)=\left\{\begin{array}{cc}
2 \bar{F}_{R}(n), & n: \text { odd }, \\
O, & n: \text { even }
\end{array}\right.
\end{gathered}
$$

Further, due to the internal symmetries of $Q_{n}^{(m)}$ and $R_{n}^{(m)}$ given by 17$), F_{R}(n)$ and $\bar{F}_{R}(n)$ for odd $n$ are expressed as

$$
F_{R}(n)=f^{(0)}(n) \mathbb{I}+\sum_{k=1}^{2 m-1} f^{(k)}(n) e_{k}, \quad \bar{F}_{R}(n)=f^{(0)}(n) \mathbb{I}-\sum_{k=1}^{2 m-1} f^{(k)}(n) e_{k} .
$$

Here the functions $f^{(0)}(n)$ and $f^{(k)}(n)$ are real. From the asymptotic form of the Lax matrix $V_{n}$ as $n \rightarrow \pm \infty$, the time dependences of the scattering data are given by

$$
\begin{gathered}
A(z, l)=A(z, 0), \\
B(z, l) A(z, l)^{-1}=B(z, 0) A(z, 0)^{-1}\left(\frac{1-\delta t z^{2}}{1-\delta t / z^{2}}\right)^{l}, \\
C_{j}(l)=C_{j}(0)\left(\frac{1-\delta t z_{j}^{2}}{1-\delta t / z_{j}^{2}}\right)^{l} .
\end{gathered}
$$

Thus $f^{(0)}(n)$ and $f^{(k)}(n)$ satisfy the following dispersion relation:

$$
\frac{1}{\delta t}\left\{\tilde{f}^{(j)}(2 n+1)-f^{(j)}(2 n+1)\right\}=\tilde{f}^{(j)}(2 n+3)-f^{(j)}(2 n-1), \quad j=0,1, \ldots, 2 m-1 .
$$

We can solve the initial-value problem of the full-discrete CNLS equations (13) in the following steps.

(a) For given potentials at $l=0, Q_{n}^{(m)}(0)$ and $R_{n}^{(m)}(0)$, we solve the direct problem of scattering, (15), and obtain the scattering data $\left\{B(z) A(z)^{-1}, z_{j}, C_{j}\right\}$.

(b) The time dependences of the scattering data are given by (22).

(c) We substitute the time-dependent scattering data into the Gel'fand-Levitan-Marchenko equations (20) and (21). Solving the equations, we reconstruct the time-dependent potentials,

$$
Q_{n}^{(m)}(l)=-\kappa_{1}(n, n+1 ; l), \quad R_{n}^{(m)}(l)=-\bar{\kappa}_{2}(n, n+1 ; l) .
$$

This step corresponds to solving the inverse problem of scattering.

In order to obtain soliton solutions, we assume that each soliton seen in $\sum_{j}\left|q_{n}^{(j)}(l)\right|^{2}$ has a timeindependent shape. By calculating an asymptotic behavior of the tails of solitons at $n \rightarrow+\infty$, we obtain the corresponding conditions,

$$
C_{2 j-1} \bar{C}_{2 j}=\bar{C}_{2 j} C_{2 j-1}=C_{2 j} \bar{C}_{2 j-1}=\bar{C}_{2 j-1} C_{2 j}=O, \quad j=1,2, \ldots, N,
$$


on the residue matrices. For instance, the one-soliton solution of $(13)$ is computed as

$$
\begin{gathered}
q_{n}^{(i)}(l)=\operatorname{sech}\left\{2 n W+2 l a+\phi_{0}\right\} \frac{\sinh 2 W}{\sqrt{\sum_{j=1}^{m}\left(\left|\alpha_{j}\right|^{2}+\left|\beta_{j}\right|^{2}\right)}}\left[\alpha_{i} \mathrm{e}^{2 \mathrm{i}\{n(\theta-\alpha)+l(b-\beta)\}}\right. \\
\left.+\beta_{i}^{*} \mathrm{e}^{-2 \mathrm{i}\{n(\theta+\alpha)+l(b+\beta)\}}\right], \quad i=1,2, \ldots, m,
\end{gathered}
$$

where $W>0, \sum_{i=1}^{m} \alpha_{i} \beta_{i}=0$ and

$$
\begin{aligned}
\mathrm{e}^{\phi_{0}} & =\frac{\sinh 2 W}{\sqrt{\sum_{j=1}^{m}\left(\left|\alpha_{j}\right|^{2}+\left|\beta_{j}\right|^{2}\right)}}, \\
\mathrm{e}^{4 a} & =\frac{\left(1-\delta t \mathrm{e}^{-2 W-2 \mathrm{i} \theta}\right)\left(1-\delta t \mathrm{e}^{-2 W+2 \mathrm{i} \theta}\right)}{\left(1-\delta t \mathrm{e}^{2 W+2 \mathrm{i} \theta}\right)\left(1-\delta t \mathrm{e}^{2 W-2 \mathrm{i} \theta}\right)}, \\
\mathrm{e}^{4 \mathrm{i} b} & =\frac{\left(1-\delta t \mathrm{e}^{2 W-2 \mathrm{i} \theta}\right)\left(1-\delta t \mathrm{e}^{-2 W-2 \mathrm{i} \theta}\right)}{\left(1-\delta t \mathrm{e}^{2 W+2 \mathrm{i} \theta}\right)\left(1-\delta t \mathrm{e}^{-2 W+2 \mathrm{i} \theta}\right)} .
\end{aligned}
$$

In the continuum limit of time $(\delta t \rightarrow 0)$, the one-soliton solution reduces to that of the semi-discrete CNLS equations [5] due to the relations,

$$
\begin{aligned}
& a=\delta t \sinh 2 W \cos 2 \theta+O\left(\delta t^{2}\right), \\
& b=\delta t \cosh 2 W \sin 2 \theta+O\left(\delta t^{2}\right) .
\end{aligned}
$$

It is noteworthy that we can obtain the most general soliton solutions of the model since we have employed the ISM for the analysis.

Conserved quantities of the full-discrete CNLS equations (12) under the decaying boundary conditions can be computed recursively by expanding the time-independent quantity, $\log \operatorname{det} A(z)$, with respect to $1 / z$. Here we list first three conserved densities,

$$
\begin{gathered}
\log \left(1-\sum_{j} q_{n}^{(j)} r_{n}^{(j)}\right), \\
\cos 2 \alpha \sum_{j}\left(q_{n+1}^{(j)} r_{n}^{(j)}+q_{n}^{(j)} r_{n+1}^{(j)}\right)+\mathrm{i} \sin 2 \alpha \sum_{j}\left(q_{n+1}^{(j)} r_{n}^{(j)}-q_{n}^{(j)} r_{n+1}^{(j)}\right), \\
\left(1-\sum_{j} q_{n+1}^{(j)} r_{n+1}^{(j)}\right)\left\{\cos 4 \alpha \sum_{j}\left(q_{n+2}^{(j)} r_{n}^{(j)}+q_{n}^{(j)} r_{n+2}^{(j)}\right)+\mathrm{i} \sin 4 \alpha \sum_{j}\left(q_{n+2}^{(j)} r_{n}^{(j)}-q_{n}^{(j)} r_{n+2}^{(j)}\right)\right\} \\
-\frac{1}{2}\left\{\cos 2 \alpha \sum_{j}\left(q_{n+1}^{(j)} r_{n}^{(j)}+q_{n}^{(j)} r_{n+1}^{(j)}\right)+\mathrm{i} \sin 2 \alpha \sum_{j}\left(q_{n+1}^{(j)} r_{n}^{(j)}-q_{n}^{(j)} r_{n+1}^{(j)}\right)\right\}^{2} \\
+\sum_{j} q_{n+1}^{(j)} r_{n+1}^{(j)} \sum_{j} q_{n}^{(j)} r_{n}^{(j)} .
\end{gathered}
$$




\section{Concluding remarks}

We have investigated an integrable full-discretization of the coupled nonlinear Schrödinger (CNLS) equations from the point of view of the inverse scattering method (ISM) for the first time in this article. We have found a Lax pair for full-discrete CNLS equations and performed the ISM under appropriate conditions with the help of the transformation (16). As a result, we can solve the initial-value problem, and obtain soliton solutions and conserved quantities.

There are plural schemes for integrable full-discretization of one-component nonlinear Schrödinger (NLS) equation. We have some freedom to determine the linearized dispersion relation of fulldiscrete NLS equation (see [3]). However, as far as we have considered, there is no such freedom in the multi-component case, which leads us to (12) as full-discrete CNLS equations. It is to be noted that (12) can be cast into a few types of full-discrete CNLS equations by coordinate transformations and redefinitions of the auxiliary variable $j_{n}$ (see [7] for the work of Hirota, Ohta and Tsujimoto on full-discrete NLS equation).

Finally, we comment that discrete CNLS equations have been studied by using the direct method [8, 9, 10]. In [8], Hirota proposed the full-discrete coupled modified KdV equations (18) and gave a $(1+1)$-soliton solution in the two-component case. Ohta obtained an $N$-soliton solution for the semi-discrete CNLS equations in terms of Pfaffian [9]. However, the $N$-soliton solution is not a general one since the initial soliton polarizations in the solution are either parallel or orthogonal. More recently, by using another Pfaffian representation, Ohta proposed more general soliton solutions without a restriction on soliton polarizations in the full-discrete case [10]. He also obtained a Lax pair of different form in 10 by means of an alternative approach.

\section{References}

[1] S. V. Manakov: Sov. Phys. JETP 38, 248-253 (1974).

[2] M. J. Ablowitz and J. F. Ladik: J. Math. Phys. 17, 1011-1018 (1976).

[3] M. J. Ablowitz and J. F. Ladik: Stud. Appl. Math. 57, 1-12 (1977).

[4] T. Tsuchida, H. Ujino and M. Wadati: J. Math. Phys. 39, 4785-4813 (1998).

[5] T. Tsuchida, H. Ujino and M. Wadati: J. Phys. A 32, 2239-2262 (1999).

[6] T. Tsuchida: Inverse Scattering Method for the Coupled Modified KdV Equations, in Proceedings of Kyushu Symposium in 1998, Recent Advances in Soliton Theory, 10ME-S1, RIAM Kyushu University [in Japanese].

[7] N. Sasa and K. Nishinari: Discrete Nonlinear Schrödinger Equations as a Scheme for Numerical Calculation, in Proceedings of Kyushu Symposium in 1998, Recent Advances in Soliton Theory, 10ME-S1, RIAM Kyushu University [in Japanese].

[8] R. Hirota: Chaos, Solitons and Fractals 11, 77-84 (2000).

[9] Y. Ohta: Chaos, Solitons and Fractals 11, 91-95 (2000).

[10] Y. Ohta: Determinant and Pfaffian Solutions for Discrete Soliton Equations, in Proceedings of the 3rd International Interdisciplinary Meeting on Symmetries and Integrability of Difference Equations, to appear. 